Paper

\title{
Invariant patterns in a non-invertible chaotic map by the directional coloring
}

\author{
Tetsushi Ueta $^{1 a)}$ \\ ${ }^{1}$ Center for Administration of Information Technology, The University of \\ Tokushima, \\ 2-1 Minami-josanjima Tokushima, Japan \\ a) ueta@tokushima-u.ac.jp
}

Received April 3, 2012; Revised May 31, 2012; Published October 1, 2012

\begin{abstract}
We propose a visualization method called the directional coloring for chaotic attractors in planer discrete systems. A color in the hue circle is assigned to the argument determined by the current point and its $n$-th mapped point. Some unstable $n$-periodic points embedded in the chaotic attractor become visible as radiation points and they can be accurately detected by combination of this coloring and the Newton's method. For a chaotic attractor in a noninvertible map, we find out invariant patterns around the fixed point and detect its nearest unstable $n$-periodic point. The computed results of their locations show a fractal property of the system.
\end{abstract}

Key Words: directional coloring, chaos, invariant pattern, fractal

\section{Introduction}

If we obtain a good mathematical model to describe dynamical behavior of the object with appropriate ways [1], we can extract much information from nonlinear phenomena emerged in the model, such as local and global bifurcations of singular points [2], chaos [3], and synchronization of oscillations [4], and so on. While, even for a chaos observed in a low-dimensional dynamical system, many problems still remain unsolved [5]. For example, computation of locations of unstable periodic orbits embedded in the chaos, identification of saddles causing crises, relationship among manifolds of saddles and chaos are still challenging problems.

While, thanks to progression of computers, simulations of a given mathematical model is easily performed by fast numerical computations [6,7]. We can observe the chaotic behavior in a computer display quickly, in addition, examine dependency of initial values and parameters within a reasonable time. Conventionally a chaotic attractor is visualized by plotting the solution orbit into a plane as a set of dots (for continuous-time systems, taking Poincaré mapping). To include statistical/dynamical information of chaos to these dots in a color display, one tries to put different color to each dot by the location, histogram of hitting [8], Lyapunov exponents [9], and so on.

In this paper, we consider utilizing of the direction held by a current point and its $n$-time mapped point, i.e., an argument defined by these two points is interpreted as a color. This can depict a transitional orientation of an orbit within the chaotic attractor. For some numbers for $n$, we find out 
unique patterns reflected from own nonlinearity. By this method, embedded unstable periodic points in a chaos attractor become visible. Although it is difficult to distinguish the locations of unstable periodic points embedded in chaotic attractors visually by the conventional methods, the proposed method can specify them as distinguishable points. Next we consider a related visualization using the brute-force method to reduce the computation time for the directional coloring. Finally, as an application, we propose an unstable periodic point detector by using the directional coloring results. Some evidences of the fractal nature about location of periodic points. With these information, an analytic formula giving the location of periodic points is derived.

\section{Conventional visualization of chaotic attractor}

Consider a two-dimensional discrete dynamical system

$$
\boldsymbol{x}_{k+1}=\boldsymbol{f}\left(\boldsymbol{x}_{k}\right),
$$

where, $\boldsymbol{x}_{k} \in \boldsymbol{R}^{2}$ is a state, and $\boldsymbol{f}: \boldsymbol{R}^{2} \rightarrow \boldsymbol{R}^{2}$ is a nonlinear function. With an appropriate initial value $\boldsymbol{x}_{0}$, a series of the solution forms an orbit such as $\left\{\boldsymbol{x}_{0}, \boldsymbol{x}_{1}, \ldots,\right\}$. On a computer display, an appropriate local region $L \subset \boldsymbol{R}^{2}$ are mapped into a screen region $S \in I \times I$ composed by pixels, and a pixel determines the resolution of this screen. Let us adopt the top-left corner of a pixel as a representative coordinate value of the pixel. Then the orbit $\boldsymbol{x}_{k}$ can be rendered as a pixel in $S$. If the system is dissipative and chaotic, the orbit may not hit each pixel uniformly in the attractor as the system iterates. In general, the orbits tend to hit around unstable periodic points or their manifolds because of the ergodic property of the chaotic attractor. The histogram of each pixel is utilized for coloring [8] i.e., color clearly indicates the points on the attractor which are most likely to be hit during the iteration process.

Let us consider the following chaotic map as a concrete example through this paper [10-12]:

$$
\left\{\begin{array}{l}
x_{k+1}=y_{k}+a x_{k} \\
y_{k+1}=-x_{k}^{2}+b
\end{array}\right.
$$

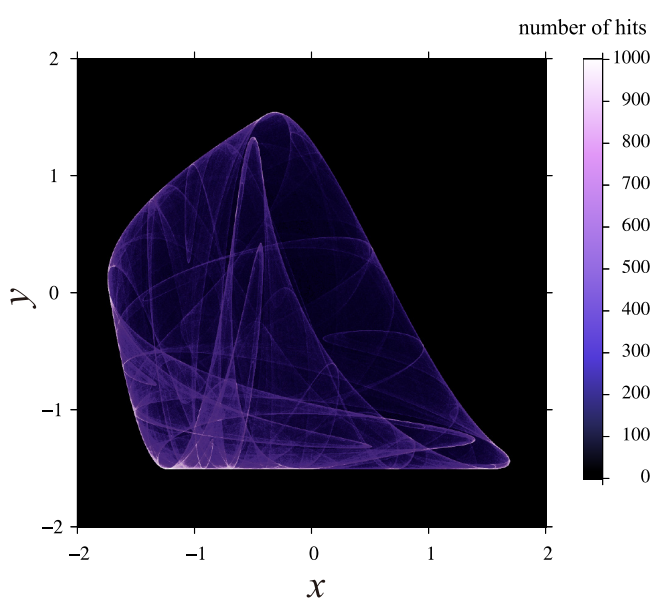

Fig. 1. Colored attractor for Eq. (2). $a=0.4, b=-1.24$.

This map is a case of the general quadratic map family [13], and depending on parameter values $a$ and $b$, it has typical bifurcations such as period-doubling, tangent, and Neimark-Sacker bifurcations. In case that $a=0.4, b=-1.24$ it gives a chaotic attractor. By using the conventional visualization mentioned above, some points colored by white are distributed along invariant sets determined by the right hand of Eq. (2). In this figure, one recognizes not only the whole structure of the strange attractor, but also an ergodic property of the orbit. This visualization, however, only shows spatial average properties of the attractor, in fact, information about the time is discarded.

\section{Directional coloring}

Now we reflect information of a movement of an orbit into the visualization. Suppose the current state is given by $\boldsymbol{x}_{k}$ and $n \geq 1$ is an integer, then measure an argument $\theta_{k}(n)$ given by $\boldsymbol{x}_{k}$ and an 
$n$-iterated point $\boldsymbol{x}_{k+n}$ in the polar coordinate. Since the argument $\theta_{k}(n)$ is $2 \pi$ modulo, it has the correspondence with the hue circle. Each iteration, evaluate $\theta_{k}(n)$ by solving Eq. (1) directly and put a corresponding color for $\theta_{k}(n)$ into $\boldsymbol{x}_{k}$. We call this color assignment method the directional coloring. Figure 2(a) shows the definition of the polar coordinate attached to $\boldsymbol{x}_{k}$ and the hue circle. With this method, a chaotic attractor is visualized like Fig. 2(b). The attractor may be segmented by several sets of colors, and any points in each of them share one direction given by $\boldsymbol{f}^{n}$, e.g., a red-colored region suggests that current points in this region will relatively move to the right after $n$ iterations.

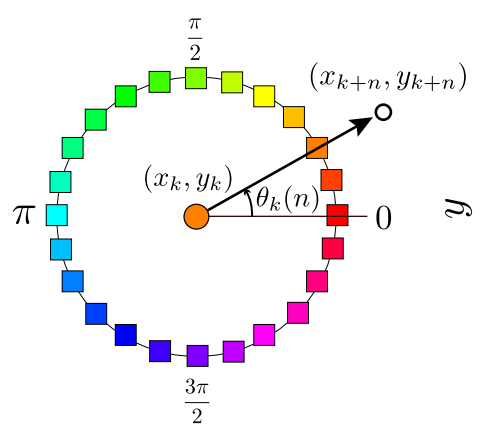

(a)

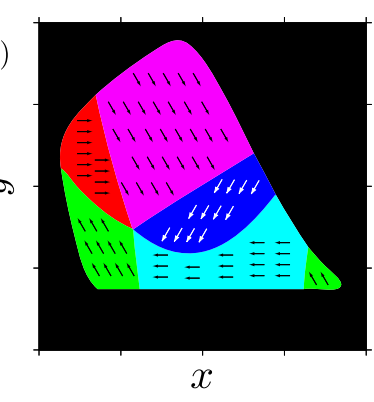

(b)

Fig. 2. Definition of the directional coloring. (a): the hue circle and the argument, (b): a rough coloring sketch. Arrows show partial directions of $\boldsymbol{f}^{n}$.

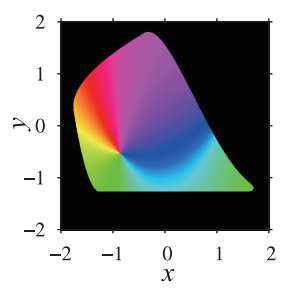

(a) $n=1$

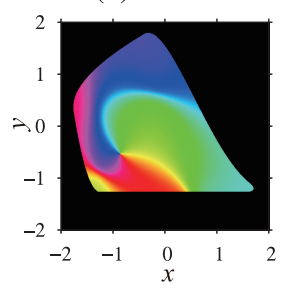

(d) $n=4$

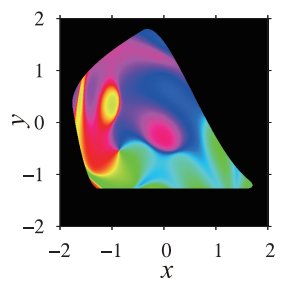

(g) $n=7$

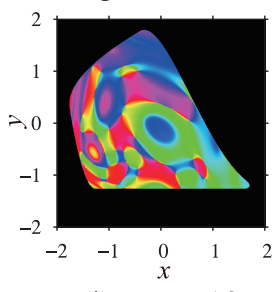

(j) $n=10$

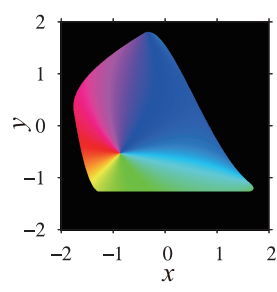

(b) $n=2$

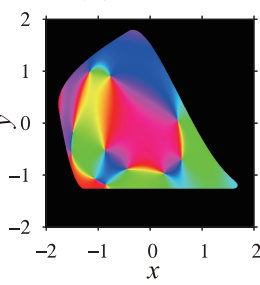

(e) $n=5$

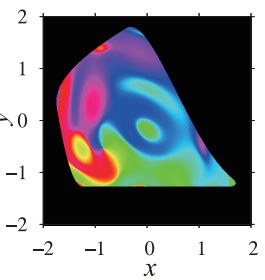

(h) $n=8$

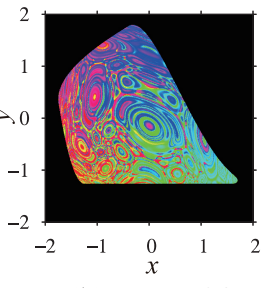

(k) $n=20$

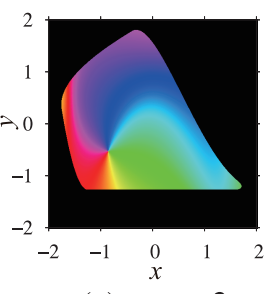

(c) $n=3$

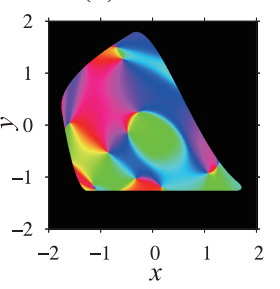

(f) $n=6$

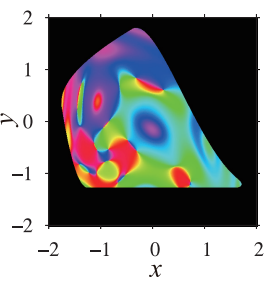

(i) $n=9$

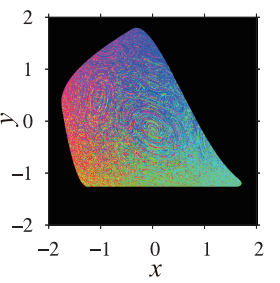

(1) $n=30$

Fig. 3. Directional coloring of the chaotic attractor observed in Eq. (2), $a=$ $0.4, b=-1.24$ with $1,000,000$ iterations. A radiation center point is clearly visible in (a)-(d). 
Figures 3 show results of the directional coloring for each $n$ iterated map written by Eq. (2) with a hundred million iterations. The parameter values are the same as ones used in Fig. 1. To retain clear visibility, we ignore the histogram information, i.e., the intensity of the color is fixed with a constant value. There are radiation points whose neighborhood forms a radial hue circle. Figure 4(a) depicts a magnification of Fig. 3(a). In the center, there is the radiation point. This point is actually a source, the location is $\boldsymbol{x}^{*} \approx(-0.85,-0.51)$, and its multipliers are $0.2 \pm i 1.29$. This source is obtained by the Neimark-Sacker bifurcation for a sink, therefore, the local space around the point retains a rotational property and causes the radial distribution of colors. One of features of the directional coloring is visualization of unstable periodic points for a specific period $n$ as a radiation point. For other types of unstable periodic points, it can be assumed that they also form such radiation points caused by changing directions of the local flows around the periodic points.

For $n \geq 5$, we have isolated single-colored islands. As $n$ increases further, the number of islands are also increased. and many $n$-periodic repellers (including repellers whose period is a common divisor of $n)$ are found as fixed points having a hue circle. Note that information of points $\boldsymbol{x}_{k+j}, j=1, \ldots, n-1$ are discarded for the coloring.

Figure 3(f) shows the case $n=20$. Many islands are confirmed in the attractor, however the macroscopical structure seems to be invariant to Fig. 3(e). It resembles the fractal process given by an iterated functional system.

In cases $n=2,3,4$, the figures are not changed much. There is a radiation point at the same position since the fixed point $\boldsymbol{x}^{*}$ can be also an $n$-periodic point. Each colored region is distorted one by one as $n$ increases. For $n=5$, new radiation points are suddenly emerged. Figure 4(b) also shows a magnification of Fig. 3(e). There are multiple radiation points indicated by arrows, in fact as discussed later, they are actually sources. More precisely, these sources are classified as unstable foci or unstable nodes, but there is no distinguishable fact between them.

A saddle type periodic point also forms a radiation point since the stable or unstable manifolds intersects at this periodic point therefore they definitely form four angles whose total is $2 \pi$. At least a local region just on the manifold moves along this manifold, thus keeps a certain direction. Thus there exists a hue circle around saddles. Although we compute transition of colors around them, there is no distinctive difference between a saddle and source as a radiation point of the directional coloring. For $n=6$, in fact, there is a tuple of 6-periodic saddle points, and its multipliers are -0.92548 and -4.59298 and they are also depicted in Fig. 3(f).

For $n=7, n=8$, we cannot recognize radiation points except for $\boldsymbol{x}^{*}$. This fact suggests that this chaos attractor does not include these periodic points. It is noteworthy that there are isolated islands filled by a single color without connecting periodic point. This is related with features of this kind of non-invertible maps $[11,12]$.

For $n=9$ to $n=20$, gradually the structure becomes complicated, but a certain fundamental pattern is unchanged, e.g, locations of some radiation points and isolated islands. This progression can be regarded as fractal. If $n$ has divisors, radiation points of them also appeared in the directional coloring. In the case of $n=20$, it can contain $2,4,5,10,20$ periodic points.

Finally $n=30$, above pattern is invisible in the large, but by ignoring small structures, the whole coloring result rather looks like the case $n=2$. This color distribution inform us a statistical prediction, e.g., many the current right-bottom (green-cyan) orbits will stay at the left-half portion after 30 iteration. For further $n>30$, this tendency retains until $n=50$, and gradually all colors are mixed. This process show a sensitivity for initial values. Similar results are obtained with the other parameter values, see Figs. 5.

With another set of parameter values; $a=-0.1, b=-1.7$, the direction coloring also exhibits distinct patterns, see Figs. 5 .

For one dimensional discrete systems $x_{k+1}=f\left(x_{k}\right)$, we visually tell where the periodic points are, indeed, the crossing points of the graph of $f^{n}$ and $x_{k+1}=x_{k}$ indicate them. Our method gives similar intuitive information about unstable periodic points in planer systems. 


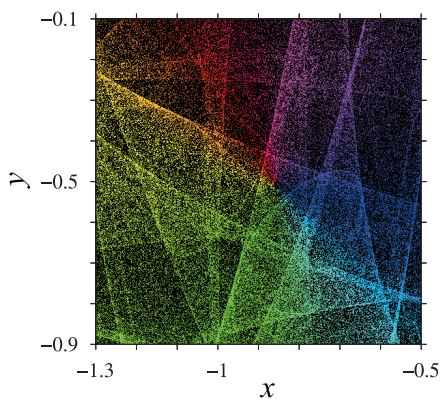

(a)

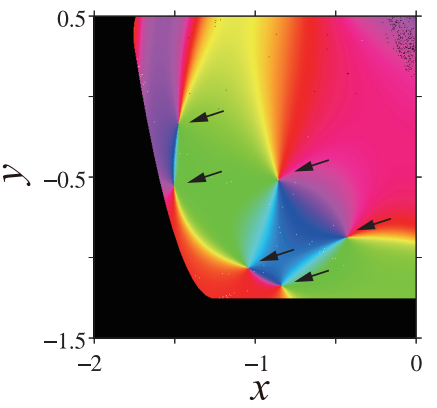

(b)

Fig. 4. Magnification of Figs. 3(a) and (e).

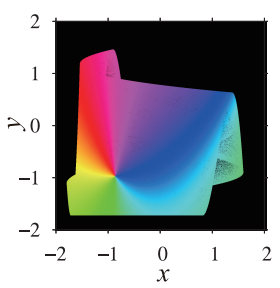

(a) $n=1$

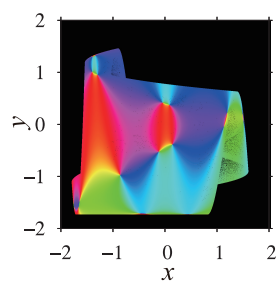

(b) $n=5$

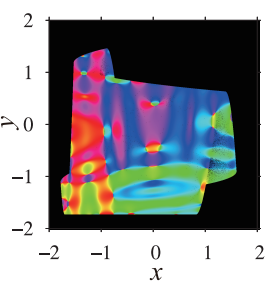

(c) $n=10$

Fig. 5. Directional coloring of the chaotic attractor observed in Eq. (2), $a=$ $-0.1, b=-1.7$.

\section{Directional coloring by brute force computation}

Figures 3 and 5 are obtained by a huge number of iterations since it cannot be expected always that the chaotic orbit hits region $S$ uniformly because of the ergodic property. To fill out a region uniformly, the computation wastes much time. In addition, if a small region inside of the attractor is selected for visualization, the computation time increases too much because the probability of hitting for such small area is rare.

Now we consider a reduction of this computation. Let us put initial points on all pixels in the region corresponding to $S$. Then evaluate the directional coloring of all pixels in $S$ with these initial points and their $n$-iterated points. Inside of a chaotic attractor region, this brute-force coloring gives a topologically same result with the coloring using solution orbits.

Figures 6(a)-(i) show coloring results by the brute-force method. The outer-shape of the strange attractor is not visualized (only in Fig. 6(a), the shape of the attractor is shown as a dashed line by an off-line work), however, if these results are trimmed along the outer-shape of the chaos attractor, the exactly same results for Figs. 3 will be obtained. Indeed, the computation time is drastically reduced because the brute-force coloring requires only $\left\{\boldsymbol{f}\left(\boldsymbol{x}_{k+1}\right), \boldsymbol{f}\left(\boldsymbol{x}_{k+2}\right), \ldots, \boldsymbol{f}\left(\boldsymbol{x}_{k+n}\right)\right\}$ are computed for each pixel. Table I shows a comparison between two methods.

It is noteworthy that some periodic points out of the chaos attractor are visualized as radiation points, e.g., a fixed point at the right-upper position in Fig. 6(a), two 2-periodic points at the leftupper and right-lower in Fig. 6(b), and so on.

Table I. Comparison with two methods. For Eq. (2), $a=0.4, b=-1.24$.

The resolution is $500 \times 500$. Computer specifications are: Intel Pentium 4,

2.6GHz, 1 GB memory, with Linux 2.4.22.

\begin{tabular}{ccc}
\hline \hline$n$ & by solutions [sec] & by brute-force [sec] \\
\hline 1 & 8.26 & 0.17 \\
2 & 9.80 & 0.21 \\
5 & 13.63 & 0.34 \\
10 & 19.76 & 0.48 \\
20 & 32.38 & 0.97 \\
\hline \hline
\end{tabular}




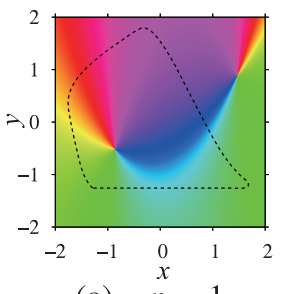

(a) $n=1$

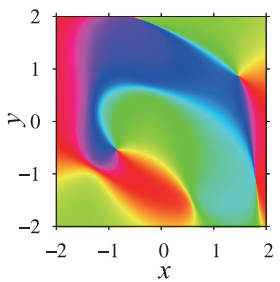

(d) $n=4$

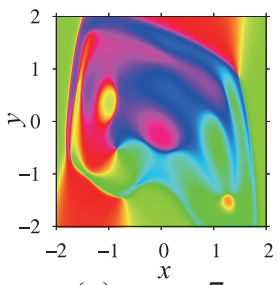

(g) $\quad n=7$

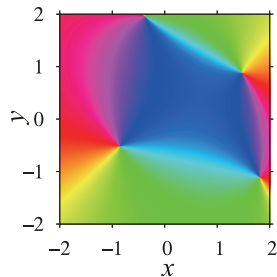

(b) $n=2$

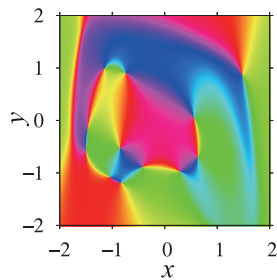

(e) $n=5$

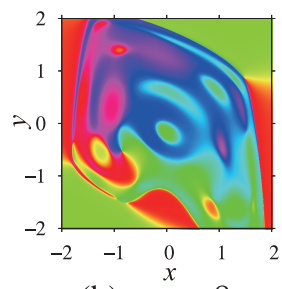

(h) $n=8$

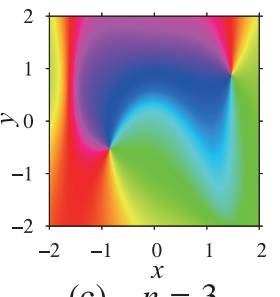

(c) $n=3$

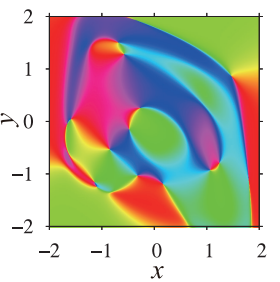

(f) $n=6$

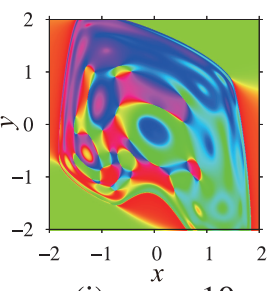

(i) $n=10$

Fig. 6. Directional coloring with the brute-force method.

\section{Unstable periodic point detector}

Theoretically, any periodic point is unstable inside a chaos attractor, i.e., any periodic point is a saddle, unstable node, or unstable focus. Detecting unstable periodic points has been studied for a long time, and many efficient methods have been proposed [14]. Literatures on recent works of detection of unstable periodic orbits in chaotic attractors are listed in [15].

As mentioned above, these periodic orbits are expressed visibly as radiation points in the directional coloring. As far as we examined, in Hénon map, and other chaotic maps, saddles embedded in a chaotic attractor can be recognized as a radiation points for a small numbers of $n$ with the directional coloring.

As an application, we propose a simple method which can detect $n$-periodic unstable periodic points in a given attractor. Let us assume any radiation point can be an unstable $n$-periodic point. For trimming of a sufficiently enlarged region $S$, firstly we seek some candidate pixels whose eight surrounding pixels form a turn in the hue circle in $S$. This scheme is a simple image processing, thus there are no technical difficulties. Next we send candidate points to Newton's method. The condition of the periodic points is:

$$
\boldsymbol{f}^{n}(\boldsymbol{x})-\boldsymbol{x}=\mathbf{0}
$$

The Jacobian matrix is obtained by solving the variational equation of Eq. (1). Newton's ensures improve accuracy of locations of the unstable periodic points from the candidates. Note that this method detects also periodic points whose period is a divisor of $n$.

Figure 7 (a) shows a complicated structure by the directional coloring for $n=37$ about the fixed point $\boldsymbol{x}^{*}$. By scaling this area, a simple structure is obtained, see, Fig. 7(b). One can easily confirm the positions of periodic points visually. The first-guess supplier also can detect candidate points accurately. In the case $n=79$, the initial guess obtained from the image is $(-0.85325626090,-0.51195375578)$, indicated as $c$ in Fig. 8. With three times iteration of Newton's method, an accurate location of 79 -periodic point $(-0.85325626092671,-0.51195375577927)$ is obtained with a $10^{-15}$ error, within 3 iterations. By iterating Eq. (2) with this solution $\boldsymbol{c}$ directly, we have the rest 78 points. They are unstable nodes since the multipliers are $3.4 \times 10^{8}$ and $-1.4 \times 10^{9}$. Furthermore, they are properly 79-periodic points since $n$ is a prime number. Note also that the repellers in this system cannot be obtained by inverse time simulation since this map is not invertible. 


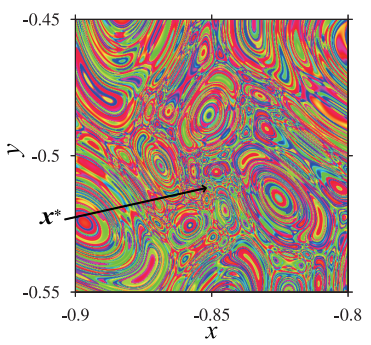

(a)

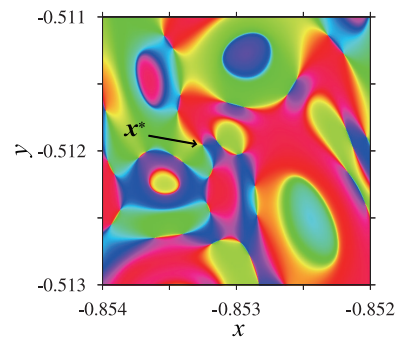

(b)

Fig. 7. Directional coloring for Eq. (2) with $n=37$. (a): around the fixed point, (b): an magnification of (a).

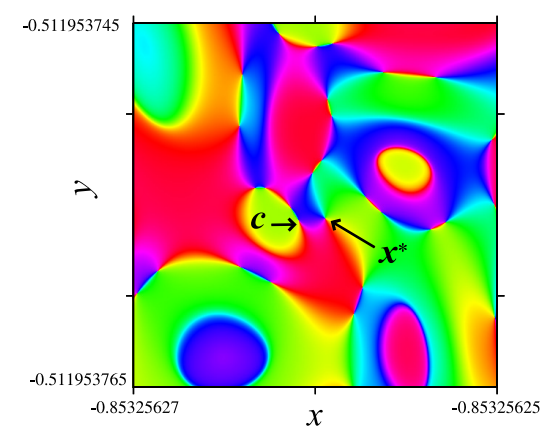

Fig. 8. Directional coloring for Eq. (2) with $n=79$. This figure shows an invariant pattern. Many unstable periodic points are visualized.

\section{Invariant pattern of fractal and locating UPPs}

One may notice that coloring patterns of Fig. 7(b) and Fig. 8 are similar, in fact, if one of them is rotated $\pi$ radian about $\boldsymbol{x}^{*}$ and rescaled a bit, their shapes are almost coincident. Thus some invariance is preserved for the directional coloring with iterations.

In this section, we model this invariance as a formula, i.e., periodic point $\boldsymbol{c}$ is expressed by a function of the number of iterations. With this formula, we could get accurate location of $\boldsymbol{c}$ without doing any shooting method. This formula features a kind of fractal nature of the map.

Let us define the distance between $\boldsymbol{c}_{k}$ and $\boldsymbol{x}^{*}$ as

$$
\epsilon_{k}=\left\|\boldsymbol{c}_{k}-\boldsymbol{x}^{*}\right\|_{2}
$$

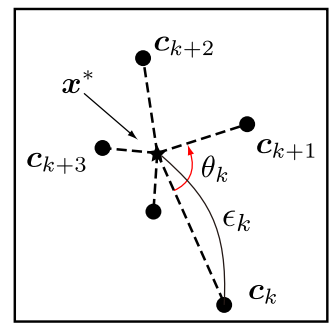

Fig. 9. The definition of $\epsilon_{k}$ and $\theta_{k}$.

Let us also define $\theta_{k}$ as an angle determined by $\boldsymbol{\epsilon}_{k+1}$ and $\boldsymbol{\epsilon}_{k}$, see Fig. 9. With our trial-and-error searching basis functions, and thanks to the the Marquardt-Levenberg algorithm (nonlinear least square estimation), we could obtain the following formulas to fit the computed values of $\epsilon$ :

$$
\tilde{\boldsymbol{c}}_{n}=\boldsymbol{x}^{*}+\sigma_{n}\left(\begin{array}{c}
\cos S \theta_{n} \\
\sin S \theta_{n}
\end{array}\right), \quad S \theta_{n}=\psi+\sum_{i=0}^{n-1} \theta_{i} .
$$

where, $\psi$ is the absolute angle between $\boldsymbol{x}^{*}$ and $\boldsymbol{c}_{1}$. Then $\sigma_{n}$ and $\theta_{n}$ are given as follows:

$$
\begin{aligned}
\sigma_{n} & =10^{a n+b+(-1)^{n} A \sin \left(\omega_{1} n-\eta_{1}\right)} \\
\theta_{n} & =(-1)^{n} B \sin \left(\omega_{2} n+\eta_{2}\right)+d
\end{aligned}
$$


These functions can specify accurate locations of UPPs $\tilde{\boldsymbol{c}}_{n}$ by only substitution of $n$.

\subsection{Example 1}

Suppose we fix the parameter values for Eq. (2) as $a=0.4, b=-1.24$, then we obtain the following parameters by using fitting method: $a=-0.1156, b=0.4, A=0.07, \omega_{1}=0.312, \eta_{1}=0.16 \pi$ and $B=0.31, \omega_{2}=0.306, \eta_{2}=-0.572 \pi, d=1.418$. Figure 10 shows matching of $\tilde{\boldsymbol{c}}_{k}$ and $\boldsymbol{c}_{k}$. The error between them is invisible.

It it noteworthy that the frequency components $\omega_{1}$ and $\omega_{2}$ are independent for the multiplier (eigenvalues) of the fixed point $\boldsymbol{x}^{*}$.

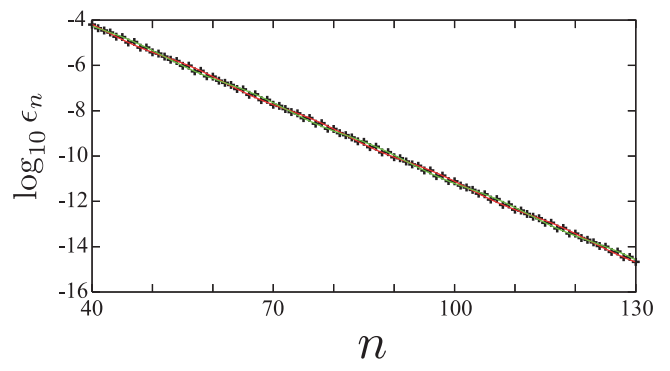

(a)

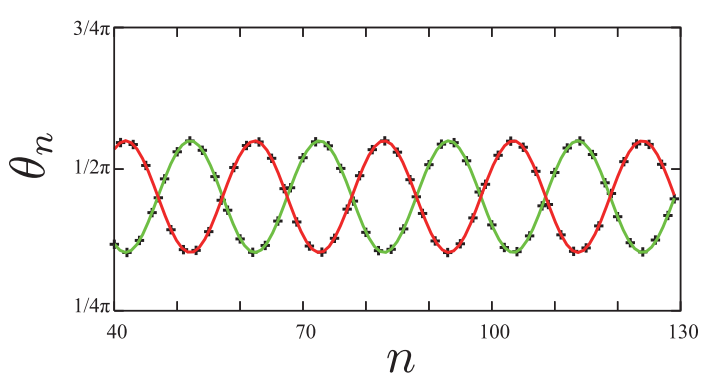

(b)

Fig. 10. Relationship between $n$ and $\epsilon_{n}$ for Example 1. Plotted points indicate numerical values of $\epsilon$.

Both $\epsilon_{k}$ and $\theta_{k}$ are affected by sinusoidal functions of $k$, but they are not synchronized each other. We once tried an amplitude modulated wave for $\epsilon_{k}$ and $\theta_{k}$ models, but it failed. Two alternative (anti-phase) sinusoidal functions are essential.

Now we obtain an analytic formula that gives UPPs in the chaos attractor. If we want to know the location of $\boldsymbol{c}_{k}$, the formula resulting the accurate location with specifying only $k$ is very useful.

Figure 11 shows locations of UPPs. In this simulation, we use $\theta_{40}$ instead of $\psi$. Each vertex of the red line show the location of $\boldsymbol{c}_{k}$ whose accuracy is guaranteed by Newton's method. While vertices on the black line are locations $\tilde{\boldsymbol{c}}_{k}$. Please note that these lines do not show the part of the solution orbit, but demonstrate a fractal nature of the chaotic attractor. In fact, a line in Fig. 11 forms a logarithmic spiral given by Eq. (5), i.e., $\epsilon_{k}$ and $\theta_{k}$ surely keep a certain scale. Note also that $\psi$ in Eq. (4) is initialized by the value of $\boldsymbol{c}_{40}$ for this case.

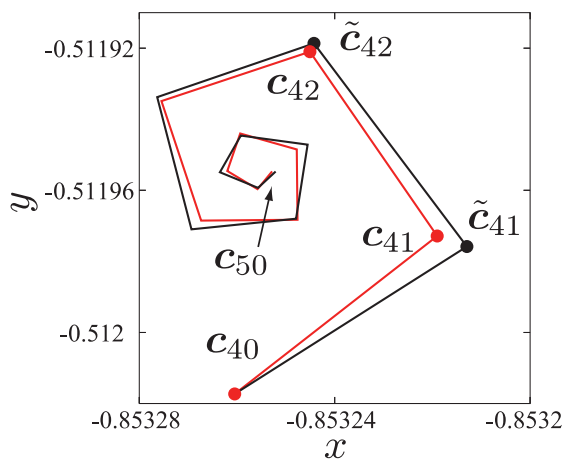

Fig. 11. $\boldsymbol{c}_{k}$ (vertices of the red line) and $\tilde{\boldsymbol{c}}_{k}$ (vertices of the black line).

\subsection{Example 2}

When we fix the parameters as $a=-0.1, b=-1.7$, a different invariant pattern of the directional coloring is shown in a chaotic attractor. Figure 13 depicts invariant patterns with $n=34$ and $n=35$ of the directional coloring around a UPP $\boldsymbol{x}^{*}=(-0.865097,-0.951606)$. The nearest radical point $\boldsymbol{c}_{n}$ is also an unstable $n$-periodic point. 


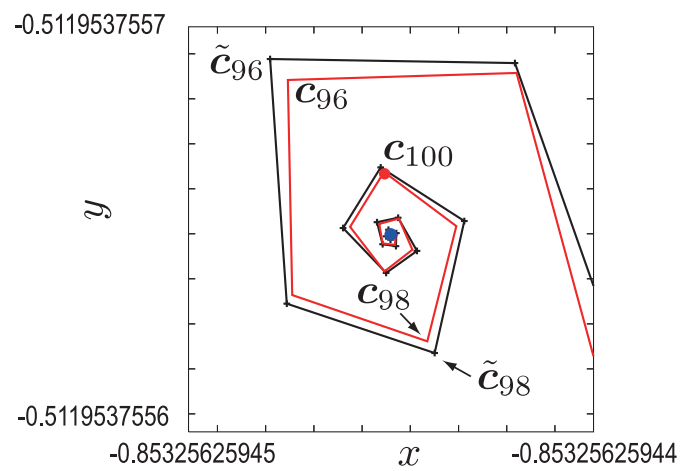

Fig. 12. An enlargement of Fig. 11. $\boldsymbol{c}_{n}, 95<n<130$ are visualized.

For this example, Eq. (5) also acts reasonable. We have a very good fitting between $\tilde{\boldsymbol{c}}_{n}$ and $\boldsymbol{c}_{n}$ with this model. The fitting parameters are as follows: $a=-0.1185, b=0.41, A=-0.07, \omega_{1}=0.08$, $\eta_{1}=0.477 \pi, B=0.27, \omega_{2}=0.08, \eta_{2}=0.0, d=1.61$.

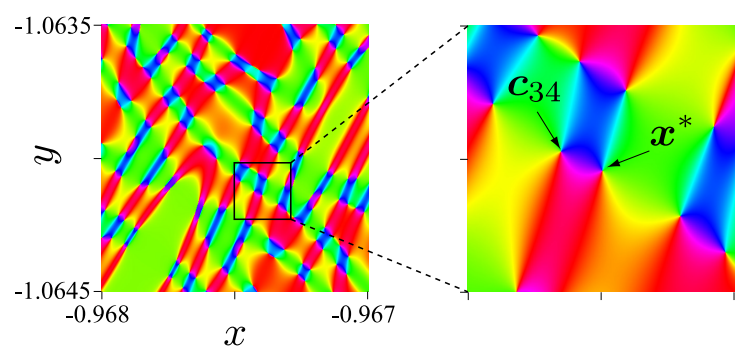

(a) $: n=34$

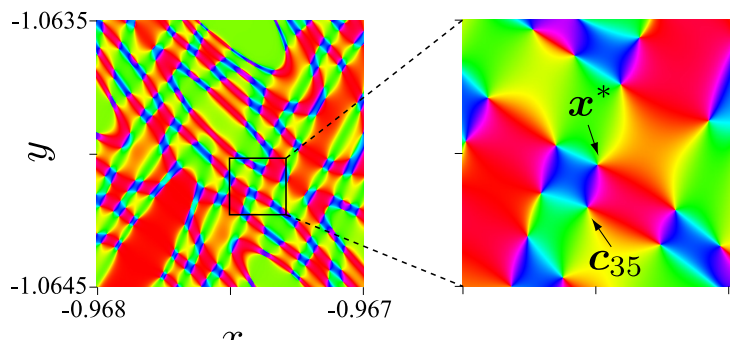

(b) $: n=35$

Fig. 13. A directional coloring result for Eq. (2) with $a=-0.1, b=-1.7$. (a): $n=34,(\mathrm{~b}): n=35$.

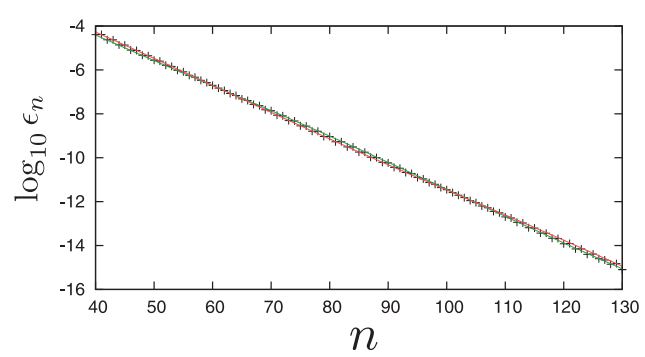

(a)

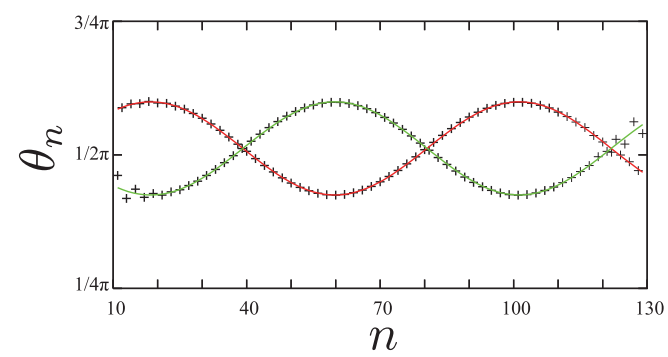

(b)

Fig. 14. Relationship between $n$ and $\epsilon_{n}$ for Example 2. Plotted points indicate numerical values of $\boldsymbol{\epsilon}$.

Figure 14 expresses agreement between $\tilde{\boldsymbol{c}}_{k}$ and $\boldsymbol{c}_{k}$. The error between them is invisible. Figure 15 shows estimation errors defined as follows:

$$
\begin{aligned}
& e_{\epsilon}=\log _{10} \epsilon_{n}-\log _{10} \tilde{\epsilon}_{n} \\
& e_{\theta}=\left|\theta_{n}\right|-\left|\tilde{\theta}_{n}\right| .
\end{aligned}
$$


where, $\tilde{\epsilon}_{n}=\left\|\tilde{\boldsymbol{c}_{n}}-\boldsymbol{x}^{*}\right\|$, and $\tilde{\theta}_{n}$ is an angle determined by $\tilde{\boldsymbol{c}}_{n}$ and $\tilde{\boldsymbol{c}}_{n+1}$. Note that $e_{\epsilon}$ shows a relative error with the logarithm scale, thus actual error between $\boldsymbol{c}_{n}$ and $\tilde{\boldsymbol{c}}_{n}$ becomes exponentially small as $n$ increases. Actually -14 in logarithm scale approaches the limitation of the double precision data length.

The mismatch between $\theta_{n}$ and $\tilde{\theta}_{n}$ can be evaluated good, that is, it is confirmed that the approximated analytic equation Eq. (5) describes almost correct locations of UPPs. The error included in $\tilde{\boldsymbol{c}}_{k}$ seems to be modeling errors in Eqs. (4) and (5), however, the oscillation behavior in Fig. 15 implies existence of one or more frequency components. We should add more sinusoidal terms in Eq. (5).

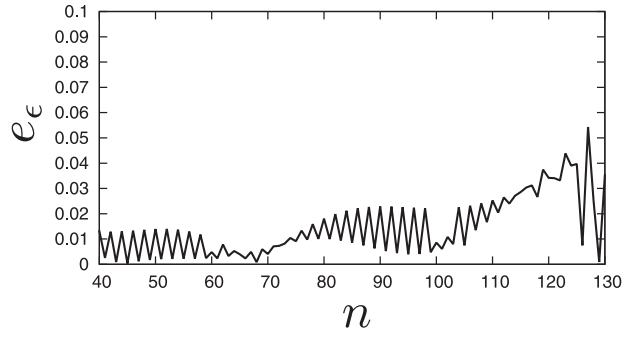

(a)

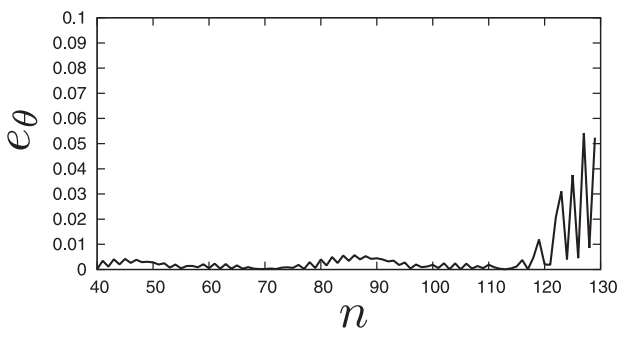

(b)

Fig. 15. Estimation errors $e_{\epsilon}$ and $e_{\theta}$.

\section{Conclusion}

We have proposed a method to visualize the direction of $\boldsymbol{f}^{n}$ inside a chaotic attractor. The method reveals an invariant pattern hidden in the chaotic attractor. With this method, UPPs with the specific number of period are visualized. As an application, a detection scheme of these UPPs is shown. Although we fix parameter values for visualization in this work, existence of $n$-periodic points, movement of locations of UPPs are confirmed by the directional coloring with changing parameters. Such information can be utilized for bifurcation analysis of UPPs. As the future works, reasons for organization of the invariant patterns should be investigated [16].

\section{Acknowledgments}

The author would like thank to T. Kumano and K. Nagao for their contributions. This work partially supported by KAKENHI 19560385.

\section{References}

[1] D. Hinrichsen and A.J. Pritchard, Mathematical Systems Theory I - Modeling, State Space Analysis, Stability and Robustness Springer Verlag, 2005.

[2] J. Guckenheimer and P. Holmes, Nonlinear Oscillations, Dynamical Systems and Bifurcations of Vector Fields, Springer Verlag, 1983.

[3] F. Moon, Chaotic and Fractal Dynamics, Springer Verlag, 1990.

[4] S.H. Strogatz, Nonlinear Dynamics and Chaos, Perseus Books, 1994.

[5] M. Benedicks and L. Carleson, "The dynamics of the Hénon map," Annals of Mathematics, vol. 133, no. 1, pp. 73-169, 1991.

[6] C.A. Pickover, Computers, Pattern, Chaos and Beauty: Graphics from an Unseen World, Dover Publications, 2001.

[7] H. Peitgen and D. Richter, The Beauty of Fractals: Images of Complex Dynamical Systems, Springer Verlag, 1986.

[8] M. Field and M. Golubitsky, Symmetry in Chaos, Oxford University Press, 1992.

[9] C. Bonatto and J.A.C. Gallas, "Accumulation horizons and period adding in optically injected semiconductor lasers," Phys. Rev. E, vol. 75, no. 055204, 2007.

[10] H. Kawakami and K. Kobayashi, "Computer experiments on chaotic situation of $x(t+2)-a x(t+$ 1) $+x^{2}(t)=b$," Bulletin of Faculty of Engineering, Tokushima University, vol. 16, pp. 29-46, 1979 . 
[11] H. Kitajima, C. Mira, and H. Kawakami, "A method to calculate basin bifurcation sets for a two-dimensional noninvertible map," International Journal of Bifurcation and Chaos, vol. 10, no. 8, pp. 2001-2014, 2000.

[12] C. Mira, D. Fournier-Prunaret, L. Gardini, H. Kawakami, and J.C. Cathala, "Basin Bifurcations of two-dimensional noninvertible maps: Fractalization of basins," International Journal of Bifurcation and Chaos, vol. 4, no. 2, pp. 343-381, 1994.

[13] J. Sprott, "Automatic Generation of Strange Attractors," Comput. E Graphics, vol. 17, pp. 325332, 1993.

[14] P. Schmelcher and F.K. Diakonos, "Detecting Unstable Periodic Orbits of Chaotic Dynamical Systems," Phys. Rev. Lett., vol. 78, no. 25, pp. 4733-4736, 1997.

[15] M. Dhamala, Y. Lai, and E.J. Kostelich, "Detecting unstable periodic orbits from transient chaotic time series," Phys. Rev. E, vol. 61, no. 6, pp. 6485-6489, 2000.

[16] C.S. Hsu, Cell-to-Cell Mapping, Springer Verlag, 1987. 